\title{
DIVULGAÇÃO CIENTÍFICA: ANALISANDO MODELOS DE COMUNICAÇÃO DA CIÊNCIA E TECNOLOGIA E IMPLICAÇÕES PARA O LETRAMENTO CIENTÍFICO E TECNOLÓGICO
}

\author{
Ruth do Nascimento Firme \\ Universidade Federal Rural de Pernambuco \\ ruthquimica.ufrpe@gmail.com \\ Priscila do Nascimento Silva \\ Universidade Federal Rural de Pernambuco \\ priscilnascimento@yahoo.com.br
}

\section{Resumo}

Neste estudo temos como objetivos identificar modelos de comunicação de ciência e tecnologia em atividades de divulgação científica, desenvolvidas através de palestras, no contexto de um projeto de extensão, em duas escolas públicas estaduais da região metropolitana do Recife-PE ao longo de 2015, e analisar implicações para o letramento científico e tecnológico dos alunos. Para análise dos resultados, selecionamos as palestras: "Ciência, Tecnologia e uma Sociedade Sustentável" e "Ciência, Tecnologia e os conflitos ambientais: o caso de Suape", e consideramos os modelos de comunicação de ciência e tecnologia como categorias analíticas. Nas análises empreendidas, identificamos predominantemente os modelos de comunicação contextual, experiência leiga e participação pública. Nessa direção, as atividades de divulgação científica se constituíram como ferramenta para o letramento científico e tecnológico dos alunos ao tempo em que as palestras contribuíram, por exemplo, para compreensão da ciência e da tecnologia como atividades humanas que influenciam e sofrem influência da sociedade.

Palavras-chave: Desenvolvimento Técnico-Científico. Questões Socioambientais. Participação Pública.

\section{SCIENTIFIC DIVULGATION: ANALYSING COMMUNICATION MODELS OF SCIENCE AND TECHNOLOGY AND IMPLICATIONS FOR THE TECHNOLOGIC AND SCIENTIFIC LITERACY}

Abstract

In this paper we aim to identify communication models of science and technology in activities of scientific divulgation developed through lectures, as extension project, at two state public schools of the Recife Metropolitan Region -PE over 2015, and analyze implication for the technologic and scientific literacy of students. For the analysis of the results, we selected the lectures named "Science, technology and a sustainable society" and "Science, technology and environment conflicts: the Suape case", and we considered the communication models of science and technology as analytical categories. In the analysis conducted were predominantly identified contextual communication model, lay expertise, and public participation. In this direction, the science divulgation activities constituted as a tool for the technologic and scientific literacy of students at the time the lectures contributed, for example, to understand the science and technology as human activities that influence and suffer influences of society.

Keywords: Technical-Scientific Development. Social-Environmental Issues. Public Participation.

\section{DIVULGACIÓN CIENTÍFICA: ANALIZANDO LOS MODELOS DE COMUNICACIÓN DE LA CIENCIA Y TECNOLOGÍA Y IMPLICACIONES PARA LA ALFABETIZACIÓN CIENTIFICA Y TECNOLÓGICA}

Resumen

En este estudio nos proponemos identificar los modelos de comunicación de la ciencia y la tecnología en actividades de divulgación de la ciencia desarrolladas a través de conferencias en el contexto de un proyecto de extensión en dos escuelas públicas en el área metropolitana de Recife-PE durante 2015 y analizar implicaciones para la alfabetización científica y tecnológica de los alumnos. Para el análisis de los resultados, hemos seleccionado las conferencias "Ciencia, Tecnología y Sociedad Sostenible" y "Ciencia, Tecnología y conflictos ambientales: el caso de Suape," y hemos considerado los modelos de comunicación de ciencia y tecnología como categorías de análisis. En los análisis realizados hemos identificado predominantemente modelos de la comunicación contextual, de la experiencia leiga y de la participación pública. Así, la actividad de divulgación científica constituyese como herramienta para la alfabetización científica y tecnológica de los estudiantes mientras las conferencias ayudaron a entender la ciencia y la tecnología como actividades humanas que influyen y son influenciadas por la sociedad.

Palabras claves: Desarrollo Técnico y Científico. Cuestiones Socioambientales. Participación Pública. 
Divulgação científica: analisando modelos de comunicação da ciência e tecnologia e implicações para o letramento científico e tecnológico

\section{INTRODUÇÃO}

É inegável a contribuição que a ciência e a tecnologia trouxeram para a sociedade nos últimos tempos. Sob um olhar mais atento, podemos afirmar que hoje, mais do que nunca, está estabelecida uma relação marcadamente profunda entre a sociedade e os domínios do conhecimento científico e tecnológico. Nesse sentido, para Bustamante (1997, p. 12):

O papel que, hoje em dia, a ciência e a tecnologia desempenham em nossa sociedade é tão profundo e extenso que se torna difícil conceber um único âmbito de atividade em que não estejam presentes ou, ainda, não modifiquem, substancialmente, atitudes, comportamentos, formas de relação, ou não propunham novas formas de fazer, de pensar e sentir e não ponham em questão valores tradicionalmente assumidos.

Entretanto, é preciso levar em conta que "a ciência e a tecnologia não estão apenas conformando nossas vidas para melhor, mas também, em muitas situações, fazendo-as perigosas" (BAZZO, 1998, p. 127). Isso porque muitos dos problemas sociais e ambientais da atualidade são desencadeados pelo desenvolvimento científico e tecnológico, como, por exemplo, a contaminação do ar por processos industriais, dos solos pelo lixo ou substâncias sólidas perigosas e das águas pelos fluxos de líquidos contaminantes de origem industrial ou agrícola sem tratamento; a contaminação sonora associada à atividade industrial ou ao transporte; e a contaminação luminosa que afeta o repouso noturno dos seres vivos alterando seus ciclos vitais (GIL PÉREZ; VILCHES, 2003).

Portanto, são estas e outras situações contraditórias de possibilidades e limitações, de riscos e vantagens e de benefícios e prejuízos envolvendo a ciência e a tecnologia que nos fazem a refletir sobre o desenvolvimento científico e tecnológico e suas aplicações e implicações sociais e ambientais. Nesse contexto, considerando os problemas sociais e ambientais decorrentes das aplicações científicas e tecnológicas, podemos dizer que vivemos em uma sociedade de risco. A sociedade de risco é concebida como uma "fase da modernidade na qual as ameaças até agora produzidas no caminho da sociedade industrial começam a predominar" (BECK et al., 2000, p. 5). Em outras palavras, podemos considerar como sociedade de risco aquela que "surge na continuidade dos processos de modernização automatizada, que são cegos e surdos aos seus próprios efeitos e ameaças" (BECK et al., 2000, p. 3).

Adicionalmente, vivemos em uma sociedade de risco ao tempo em que os problemas sociais e ambientais desta sociedade: geram um sentimento de perigo e ameaça; não são limitados por fronteiras; têm consequências são incalculáveis, em muitos casos, incompensáveis; e não 
Divulgação científica: analisando modelos de comunicação da ciência e tecnologia e implicações para o letramento científico e tecnológico

podem ser identificados em um período de tempo definido (BECK, 2011). Portanto, sociedade de risco é um modelo de sociedade no qual nos sentimos colocados em risco pelo próprio desenvolvimento científico e tecnológico (RESENDE; ROTHBERG, 2011).

É considerando o conceito de sociedade de risco que destacamos a necessidade da formação de cidadãos científica e tecnologicamente letrados capazes de interagirem com os paradoxos da modernidade e tomarem decisões responsáveis quanto às aplicações e implicações da ciência e tecnologia na sociedade. O letramento científico e tecnológico é compreendido como o "estado ou a condição de quem não apenas conhece a linguagem científica e entende alguns de seus princípios básicos, mas exerce práticas sociais que usam o conhecimento científico e tecnológico" (SANTOS, 2006, p. 613).

Adicionalmente, com base nas ideias de Fourez (1994), assumimos que um cidadão científica e tecnologicamente letrado é capaz de, por exemplo, utilizar conceitos científicos, integrar valores e tomar decisões, compreender que a sociedade exerce e sofre influências do desenvolvimento científico e tecnológico, reconhecer que o conhecimento científico é provisório e sujeito a mudanças, e conhecer fontes válidas de informação científica e tecnológica recorrendo a elas diante de situações de tomada de decisão.

Portanto, o que se pretende com letramento científico e tecnológico é a ampliação das possibilidades de participação de cidadãos visando o exercício de sua cidadania e a formação de uma sociedade mais democrática quanto ao poder de decisão sobre aplicações e implicações da ciência e tecnologia na sociedade, visto que "a participação dos cidadãos na tomada de decisões é hoje [...], uma garantia de aplicação do princípio de precaução, que se apóia numa crescente sensibilidade social face às implicações do desenvolvimento técnico-científico [...]" (CACHAPUZ et al., 2005, p. 28).

Nesse sentido, em uma sociedade de risco, o acesso à informação e ao letramento científico e tecnológico dos indivíduos parece ser um caminho estratégico para propiciar a participação dos cidadãos na tomada de decisões frente às implicações sociais e ambientais das aplicações da ciência e da tecnologia na sociedade.

Mas, como promover o acesso à informação? Como promover o letramento científico e tecnológico dos cidadãos? Quais os caminhos possíveis para que cidadãos comuns tenham acesso a informações e discussões sobre temáticas atuais envolvendo, por exemplo, sustentabilidade, conflitos ambientais, controvérsias científico-tecnológicas, relação custo/benefício das inovações tecnológicas, etc. que gerem reflexões, questionamentos e o desenvolvimento de uma postura de participação cidadã? Como oportunizar um espaço que incentive o debate sobre as aplicações e 
Divulgação científica: analisando modelos de comunicação da ciência e tecnologia e implicações para o letramento científico e tecnológico

implicações do desenvolvimento científico e tecnológico na sociedade e aproxime os cidadãos de conhecimentos da ciencia e da tecnologia que interferem no cotidiano dos mesmos? Buscando respostas para as questões colocadas, assumimos que um dos caminhos pode ser a divulgação científica.

$\mathrm{Na}$ literatura há diferentes concepções de divulgação científica, que dependendo do autor, podem se aproximar ou não das concepções de popularização e de disseminação da ciência. Para situar o que estamos considerando como divulgação científica neste trabalho, tomamos por base a categorização proposta por Baumgarten (2012, p. 89), segundo a qual:

\footnotetext{
Divulgação de ciência e tecnologia é um processo que reflete a construção dos conhecimentos, seus embates, diálogos e necessárias composições com o conhecimento social (ou prático) para o melhor direcionamento do desenvolvimento da sociedade em seus segmentos plurais. A comunicação e divulgação científica envolvem [...] não apenas a tradução da linguagem científica para o público leigo (popularização) ou disseminação entre as coletividades científicas de conhecimentos (difusão) específicos do próprio campo. (Grifos nossos).
}

Portanto, assumimos a divulgação científica, seguindo a concepção de Baumgarten (2012), como caminho estratégico para propiciar a participação dos alunos em discussões acerca de implicações sociais e ambientais das aplicações da ciência e da tecnologia na sociedade.

Adicionalmente, Ferreira e Queiroz (2012), por exemplo, quando fizeram uma revisão da literatura sobre o uso de textos de divulgação científica no ensino de ciências, identificaram alguns objetivos destacados pelos autores dos trabalhos analisados. Entre os objetivos, estão: fomentar hábitos de leitura no contexto escolar, favorecer a compreensão sobre a construção do conhecimento científico, promover o interesse dos alunos em sala de aula, estimular o pensamento crítico, fomentar discussões e debates em sala de aula, e desenvolver habilidades de comunicação oral e escrita dos estudantes. Contudo, empreender a divulgação científica como ferramenta para o letramento científico e tecnológico não foi um objetivo identificado no conjunto dos trabalhos analisados.

Nesse contexto, partimos do pressuposto de que a divulgação científica pode se constituir em uma ferramenta para o letramento científico e tecnológico. Entretanto, precisamos considerar que existem diferentes configurações comunicativas que vão interferir no discernimento dos cidadãos na discussão de temas que envolvem o desenvolvimento científico e tecnológico. Nesta direção, este estudo é conduzido a partir da seguinte questão de pesquisa: quais modelos de comunicação de ciência e tecnologia estão presentes em atividades de divulgação 
Divulgação científica: analisando modelos de comunicação da ciência e tecnologia e implicações para o letramento científico e tecnológico

científica e quais implicações desses modelos para o letramento científico e tecnológico de cidadãos comuns?

$\mathrm{Na}$ tentativa de buscar respostas para a questão de pesquisa proposta, discutiremos diferentes modelos de comunicação da ciencia e tecnologia. Nessa direção, segundo Fares, Navas e Marandino (2007), quatro modelos de comunicação de ciencia e tecnologia podem ser considerados: o modelo de déficit cognitivo; o modelo contextual; o modelo da experiência leiga; e o modelo de participação pública.

No modelo de déficit cognitivo, o processo de comunicação se dá em uma única via e considera os cientistas como emissores, especialistas e possuidores do conhecimento e os cidadãos comuns como receptores passivos com déficit de conhecimentos sobre a ciência e a tecnologia (FARES; NAVAS; MARANDINO, 2007). Segundo Baumgarten (2012), neste modelo de comunicação há um entendimento de uma sociedade dividida entre especialistas e leigos. Desta forma, este tipo de modelo de comunicação considera "que a transmissão do conhecimento eliminaria, de maneira automática, a defasagem entre especialistas e leigos" (RESENDE; ROTHBERG, 2011, p. 52).

No modelo contextual, o processo de comunicação ainda se dá em uma única via emissor (cientista)/receptor (público), entretanto, são valorizadas as experiencias culturais, os conhecimentos prévios e o contexto dos cidadãos comuns reconhecendo que a compreensão desses últimos é facilitada quando os conhecimentos da ciência e da tecnologia fazem parte de seus contextos (FARES; NAVAS; MARANDINO, 2007). Nesse sentido, no modelo contextual é esperado que as informações sobre a ciência e a tecnologia sejam discutidas considerando os contextos "social, econômico e político" nos quais as atividades científicas e tecnológicas estão inseridas (BAUMGARTEN, 2012, p. 90).

Em contrapartida, o modelo da experiência leiga caracteriza-se como um modelo de comunicação dialógico que valoriza os conhecimentos locais considerando-os tão importantes quanto os conhecimentos científicos, para a resolução de problemas (FARES; NAVAS; MARANDINO, 2007). Portanto, este modelo de comunicação é considerado mais democrático quando comparado aos anteriormente discutidos, visto que questiona "a superioridade do saber científico e tende a eliminar a ideia de déficit, pois admite que o conhecimento científico seja formado tanto pelas informações especializadas quanto por aquelas adquiridas pela experiência" (RESENDE; ROTHBERG, 2011, p. 54).

Por fim, o modelo de participação pública, baseado em uma perspectiva dialógica, é um modelo de comunicação que valoriza o diálogo entre cientista e não cientista e busca a 
Divulgação científica: analisando modelos de comunicação da ciência e tecnologia e implicações para o letramento científico e tecnológico

democratização da ciência e da tecnologia através da participação dos cidadãos em espaços propícios como foros, debates e conferencias de consenso (FARES; NAVAS; MARANDINO, 2007). Segundo Resende e Rothberg (2011), o modelo de participação pública pode contribuir tanto para ampliar o controle das decisões sobre ciência e tecnologia como para aumentar o diálogo entre a elite política e científica e os cidadãos através do exercício de participação pública visando à formação de consensos razoáveis.

Portanto, considerando a importância do letramento científico e tecnológico dos cidadãos em uma sociedade de risco e o papel da divulgação científica nesse processo, temos como objetivos neste estudo: identificar modelos de comunicação de ciência e tecnologia em atividades de divulgação científica desenvolvidas em escolas públicas da Região Metropolitana do Recife-PE e analisar implicações para o letramento científico e tecnológico dos alunos.

\section{MATERIAIS E MÉTODOS}

Este estudo emerge no contexto do projeto de extensão "Divulgação Científica em Escolas Públicas: uma ferramenta para o letramento científico e tecnológico", apoiado pela PróReitoria de Atividades de Extensão (PRAE) da Universidade Federal Rural de Pernambuco (UFRPE), e desenvolvido em duas escolas públicas estaduais da região metropolitana do Recife$\mathrm{PE}$, com o objetivo promover o letramento científico e tecnológico e o desenvolvimento de reflexão crítica e de uma postura de participação aos estudantes do ensino médio através da divulgação científica.

O respectivo projeto de extensão foi executado de janeiro a dezembro de 2015 e contemplou o seguinte conjunto de ações: (1) estudo e discussão sobre as temáticas das palestras; (2) discussão e detalhamento do plano de trabalho do aluno bolsista; (3) visitação às escolas para a apresentação do projeto de extensão; (4) visitação às escolas para definição do cronograma das palestras; (5) organização de material para a elaboração das palestras; (6) apresentação mensal das palestras nas escolas; (7) construção de um blog; elaboração de questionários; (8) escrita de trabalhos para participação em congressos; (9) eventos e seminários de atividades de extensão; (10) elaboração de relatórios parciais e final; (11) e avaliação do projeto de extensão.

Uma das ações do projeto de extensão foi a construção de um blog para ampliar o espaço de discussão sobre as temáticas tratadas nas palestras no contexto escolar. Nesse sentido, com o blog visamos atender aos objetivos de possibilitar aos alunos das escolas parceiras, e também aos 
Divulgação científica: analisando modelos de comunicação da ciência e tecnologia e implicações para o letramento científico e tecnológico

seus professores, a postagem de comentários e/ou de respostas aos questionamentos lançados quando as palestras são proferidas nas escolas, e o de propiciar à equipe executora a postagem de vídeos, artigos, enquetes, notícias, cronograma das palestras, etc.

Neste projeto de extensão, a atividade de divulgação científica foi realizada através de 08 palestras mensais, de julho a novembro, apresentadas nas escolas em turmas de Ensino Fundamental e Médio abordando as temáticas a seguir: Ciência, tecnologia e uma sociedade sustentável (Palestra 1); Ciência, tecnologia e os conflitos ambientais: o caso de Suape (Palestra 2); Ciência, tecnologia e as políticas públicas (Palestra 3); Inovação tecnocientíficas e suas controvérsias (Palestra 4); A participação pública no contexto da ciência e da tecnologia (Palestra 5); A energia nuclear no Brasil: qual a relação risco/benefício? (Palestra 6); Lixo e a destinação de resíduos sólidos (Palestra 7); O desenvolvimento científico-tecnológico em Suape (Palestra 8).

As palestras foram apresentadas em data show e elaboradas e organizadas em slides no programa PowerPoint ${ }^{\circledR}$. Cada palestra teve duração, em média, de 50 minutos e seguiu um percurso metodológico dependente da temática abordada. Por exemplo, em algumas palestras fez-se a inserção de um debate, em outras foram aplicados questionários e em outras foram colocados questionamentos para os alunos responderem no blog do projeto de extensão.

Considerando que a divulgação científica foi realizada através de palestras, neste estudo específico analisamos duas das oito palestras apresentadas ao longo do desenvolvimento do projeto de extensão. Para a seleção das duas palestras analisadas consideramos uma palestra cuja temática abordada foi mais geral, neste caso a Palestra 1 - Ciência, tecnologia e uma sociedade sustentável, e outra com uma temática mais específica do contexto dos pernambucanos, neste caso a Palestra 2 - Ciência, tecnologia e os conflitos ambientais: o caso de Suape.

Portanto, o movimento analítico das palestras selecionadas contemplou, inicialmente, a descrição do desenvolvimento das mesmas e, em seguida, a análise propriamente dita considerando como categorias analíticas os modelos de comunicação de ciência e tecnologia (FARES; NAVAS; MARANDINO, 2007) discutidos anteriormente, a saber: modelo de déficit cognitivo, o modelo contextual, o modelo da experiência leiga e o modelo de participação pública. Posteriormente, buscamos analisar implicações dos modos de comunicação de ciência e tecnologia para o letramento científico e tecnológico dos alunos. 
Divulgação científica: analisando modelos de comunicação da ciência e tecnologia e implicações para o letramento científico e tecnológico

\section{RESULTADOS E ANÁLISES}

A palestra 1, composta por 19 slides, teve início com o seguinte questionamento, conforme figura 1: Vamos entender primeiro o que sustentabilidade. Você sabe o que é?

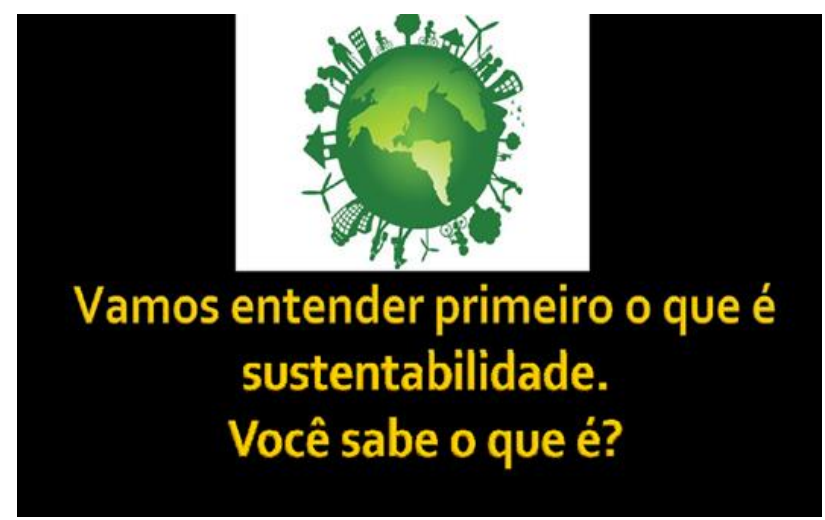

Figura 1: Terceiro slide da palestra 01 - Ciência, tecnologia e uma sociedade sustentável, realizada em escolas públicas do Ensino Médio de Recife (PE), no ano de 2015.

Fonte: Elaborada pelo palestrante

Nesse sentido, após a apresentação deste questionamento aos alunos, foram discutidos alguns significados da palavra sustentabilidade propostos no dicionário. Em seguida, foram lançadas as seguintes questões para os alunos: quanto mais desenvolvimento menos sustentabilidade? A solução para o problema seria parar de desenvolver? O desenvolvimento é bom ou não?

Após as discussões sobre estas questões com os alunos, foi apresentado o vídeo "A estória das coisas". Este vídeo propõe uma discussão crítica sobre um modelo de desenvolvimento em um sistema capitalista que gera um comportamento de consumo pelas pessoas, na maioria das vezes, desnecessário. Dessa forma, o objetivo da apresentação deste vídeo na palestra 1 foi exatamente trazer para discussão a necessidade de se refletir sobre o consumo desenfreado e suas consequências. Como por exemplo, a destinação dos resíduos de produção, a valorização do consumo em um sistema capitalista, a cultura do ter sem precisar, etc, e destacar a ideia de um desenvolvimento sustentável.

Seguida da exibição do vídeo 1, foi iniciada uma discussão sobre a inegável contribuição da ciência e da tecnologia nos últimos anos para a sociedade e, ao mesmo tempo, sobre a necessidade de não se confiar excessivamente nelas, tornando-nos cegos pelo conforto que nos 
Divulgação científica: analisando modelos de comunicação da ciência e tecnologia e implicações para o letramento científico e tecnológico

proporcionam cotidianamente através do uso de seus aparatos e dispositivos técnicos. Nessa direção, foram lançadas aos alunos as seguintes questões: Quais os benefícios do desenvolvimento científico e tecnológico? Quais os malefícios? Que atitudes podem ajudar a minimizar impactos deste desenvolvimento na sociedade e no ambiente?

Posteriormente, foram colocados para os alunos alguns dados do INEP sobre o consumismo e, em seguida, apresentado o vídeo 2 intitulado "Rio+20: Desafios da sustentabilidade". Este vídeo traz uma discussão sobre o desenvolvimento e suas relações com o uso dos recursos naturais. Portanto, este segundo vídeo foi apresentado com o objetivo de fazer os alunos refletirem e se posicionarem sobre a relação custo/benefício relativa ao uso dos recursos naturais.

Após a exibição do vídeo 2, foi apresentada aos alunos uma imagem (Figura 2) com a seguinte pergunta: o que há de errado nesta imagem?

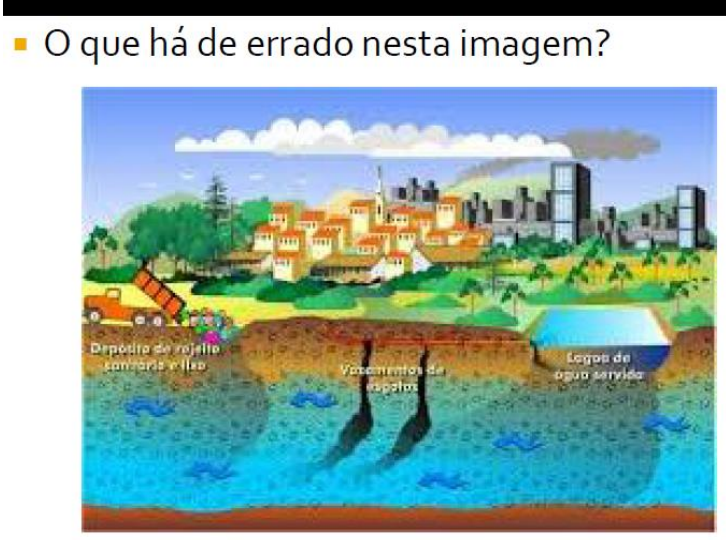

Figura 2: Décimo segundo slide da palestra 01 - Ciência, tecnologia e uma sociedade sustentável, realizada em escolas públicas do Ensino Médio de Recife (PE), no ano de 2015.

Fonte: Ache tudo e região

Disponível em: https://www.achetudoeregiao.com.br/

A partir da pergunta colocada, foram discutidas com os alunos questões referentes: ao quadro atual de degradação e riscos provocados por estilos de vida e de produção incompatíveis com a permanência dos recursos naturais; à sustentabilidade como o principal desafio para o desenvolvimento social e; ao desafio de caráter político, além de técnico, de se buscar por um modelo de desenvolvimento sustentável, visto que estes são alguns dos âmbitos que estão à frente dos processos decisórios em vários campos.

Posteriormente, voltou-se a proposição da sustentabilidade como resultado das escolhas sobre as formas de produção, consumo, habitação, comunicação, alimentação, transporte e 
Divulgação científica: analisando modelos de comunicação da ciência e tecnologia e implicações para o letramento científico e tecnológico

também nos relacionamentos entre as pessoas e delas com o ambiente, considerando os valores éticos, solidários e democráticos. Na sequência, foram discutidos os pilares da sustentabilidade (social, ambiental e econômico) e algumas atitudes sustentáveis que podem ser tomadas no cotidiano, destacando o desenvolvimento sustentável como um modelo de desenvolvimento.

Por fim, após as discussões mencionadas anteriormente, nos dois últimos slides da palestra 1 foram lançados uma imagem (Figura 3) e o seguinte questionamento aos alunos: $\mathrm{O}$ que você pode fazer para que num futuro próximo a mensagem dessa figura não seja uma realidade? Comenta lá no BLOG!

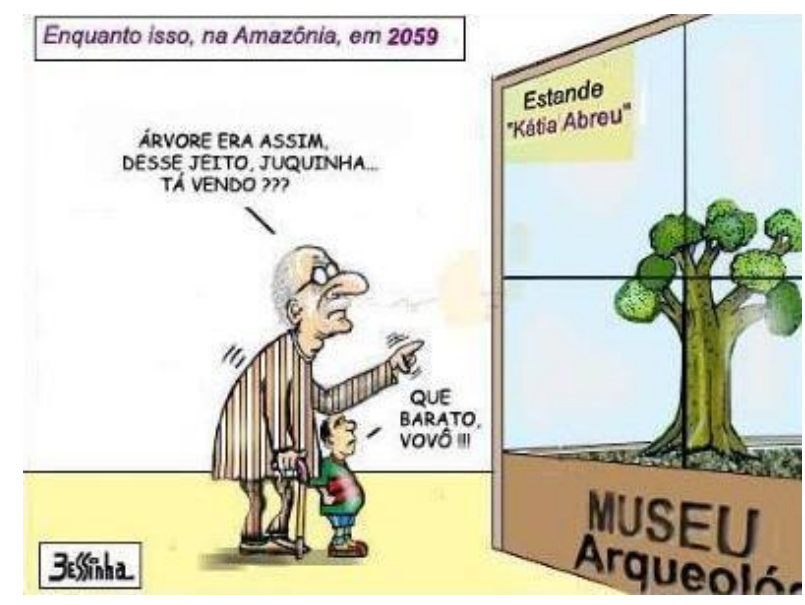

Figura 3: Décimo nono slide da palestra 01 - Ciência, tecnologia e uma sociedade sustentável, realizada em escolas públicas do Ensino Médio de Recife (PE), no ano de 2015. Fonte: BESSINHA.

Disponível em: https://pattindica.wordpress.com/2009/06/16/charge-do-bessinha-2059-enquanto-isso-naamazonia/

A palestra 2 foi composta por 27 slides e teve início com uma discussão histórica sobre o funcionamento do porto de Suape. Em seguida, a palestrante iniciou uma discussão sobre o Complexo de Suape abordando aspectos, como, por exemplo, estatísticas de importação e exportação, os municípios que este complexo abrange etc. Posteriormente, foram apresentadas algumas fotos do complexo de Suape.

Após apresentação das fotos, os alunos assistiram ao vídeo intitulado "Vídeo Institucional sobre o Porto de Suape”. Este vídeo discute o que é o Portuário de Suape e justifica a escolha da área geográfica, por ser um ponto estratégico para embarque e desembarque das grandes navegações, e destaca sua importância para as cidades circunvizinhas. Portanto, este vídeo foi apresentado na palestra 2 com o objetivo de discutir com os alunos aspectos positivos e negativos relativos às atividades deste Portuário, visto que, de fato, é um grande empreendimento 
Divulgação científica: analisando modelos de comunicação da ciência e tecnologia e implicações para o letramento científico e tecnológico

para o Estado de Pernambuco, pois é uma fonte geradora de muitos empregos e desenvolvimento. Após a apresentação do vídeo, foram apresentadas as empresas que fazem parte do Complexo de Suape. Posteriormente, iniciou-se uma discussão sobre as contribuições trazidas pelo Complexo de Suape ao Estado de Pernambuco e os conflitos ambientais decorrentes de sua instalação e funcionamento.

Nesse sentido, a discussão teve início com um esclarecimento sobre o que é conflito socioambiental. Para isso foi considerado por conflito socioambiental um conflito social relacionado ao modo de apropriação e uso dos elementos da natureza envolvendo relações de poder em que os sujeitos envolvidos constroem uma dimensão ambiental para suas lutas (ACSELRAD, 1995 apud SILVEIRA; BARROS, 2013).

Dessa forma, foram discutidos alguns aspectos da evolução do homem em sua relação com natureza enquanto fonte de recursos e algumas implicações deste processo, como, por exemplo, a urbanização, o desmatamento, etc. Nessa direção, foi iniciada uma discussão sobre a visão da natureza como um "conjunto de recursos econômicos que as culturas humanas transformam em seu processo de sobrevivência e de produção" (HERCULANO, 2013, p. 8). Neste momento, foi destacado que o homem, na maioria das vezes, só usa os recursos da natureza sem se preocupar com as consequências deste comportamento.

Após a discussão sobre implicações ambientais causadas pela forma como o homem usufrui dos recursos naturais, foi apresentado aos alunos um segundo vídeo. O vídeo 2 , intitulado "O homem capitalista", propõe uma discussão crítica sobre o capitalismo e sobre sua influência na vida das pessoas. Nesse sentido, o uso deste vídeo na palestra 2 teve como objetivo promover uma discussão crítica com os alunos sobre a relação risco/benefício do desenvolvimento científico e tecnológico numa perspectiva capitalista.

Após a apresentação do vídeo, a discussão buscou destacar problemas ambientais que o consumo desenfreado dos recursos naturais tem gerado, estando relacionado à, por exemplo, maximização dos espaços industriais. Contudo, a palestra 2 também contemplou o fato de que o Complexo de Suape também tem se preocupado com a sustentabilidade. E neste sentido, foram apresentados alguns de seus programas, como o Projeto Suape para Todos.

Por fim, foi lançado para os alunos o seguinte questionamento: Qual a relação risco/benefício dos avanços científicos e tecnológicos em SUAPE?

Após a descrição das palestras, iniciamos a análise das mesmas. Nesse sentido, quanto aos modelos de comunicação da ciência e tecnologia, identificamos aspectos do modelo de comunicação déficit cognitivo (FARES; NAVAS; MARANDINO, 2007) nas palestras 1 e 2, 
Divulgação científica: analisando modelos de comunicação da ciência e tecnologia e implicações para o letramento científico e tecnológico

quando levamos em conta que uma das intenções do palestrante era o de divulgar para os alunos conhecimentos sobre a necessidade de um modelo de desenvolvimento sustentável (Palestra 1) e sobre a relação entre o modelo de desenvolvimento capitalista e os conflitos ambientais decorrentes desse desenvolvimento (Palestra 2), os quais, a palestrante considerava que eles não tinham até o momento das palestras.

Entretanto, a continuidade da análise das palestras 1 e 2 nos permitiu identificar a predominância dos outros modelos de comunicação da ciência e da tecnologia em detrimento do modelo de comunicação déficit cognitivo. Uma das preocupações visíveis da palestrante ao longo do desenvolvimento das duas palestras foi a de não considerar o público, neste caso os alunos do ensino médio, como ouvintes passivos. E este fato configura um distanciamento do modelo de comunicação déficit cognitivo. A palestra 1 foi iniciada efetivamente perguntando se os alunos sabem o que sustentabilidade. Adicionalmente, a proposição da atividade de análise da imagem (Figura 2) pelos alunos na palestra 1 e as apresentações e discussão dos vídeos nas palestras 1 e 2 são evidências da busca da palestrante por uma maior interação com os alunos frente as temáticas abordadas.

Além disso, entendemos que em ambas as palestras, a visão de ciência e de tecnologia como motoras de progresso, ou como formas de conhecimentos que são construídas sem influenciar ou sofrer influências da sociedade, não foi privilegiada. Pelo contrário, buscou-se evidenciar uma visão da atividade científica e tecnológica em sua relação com o contexto socioambiental. Na palestra 1, a atividade de análise da imagem (Figura 2) pelos alunos contribuiu para explicitar relações entre ciência, tecnologia e sociedade, ou seja, relações entre aplicações científicas e tecnológicas e implicações decorrentes dessas aplicações na sociedade e no meio ambiente.

Aspectos do modelo de comunicação contextual (FARES; NAVAS; MARANDINO, 2007) foram identificados à medida que foram valorizados contextos das atividades científicas e tecnológicas discutidas. Nesse sentido, embora a temática da palestra 1 - Ciência, tecnologia e uma sociedade sustentável permeie o contexto da maioria dos cidadãos, foi na palestra 2 que se buscou discutir as informações científicas e tecnológicos em seus contextos de produção e aproximar a temática ao contexto dos alunos, considerando que o Complexo de Suape, influenciou, influencia ou influenciará a vida de muitos pernambucanos de diferentes formas, como, por exemplo, nas oportunidades de trabalho geradas neste complexo portuário.

Aspectos do modelo de comunicação da experiência leiga (FARES; NAVAS; MARANDINO, 2007) também foram identificados ao longo do desenvolvimento das duas 
Divulgação científica: analisando modelos de comunicação da ciência e tecnologia e implicações para o letramento científico e tecnológico

palestras analisadas. Ao longo da apresentação das palestras houve a intenção de questionar a superioridade do conhecimento científico e tecnológico em resolver problemas sociais em detrimento de conhecimentos éticos, políticos, econômicos e de conhecimentos construídos ao longo da experiência dos cidadãos, e isto ficou bem evidente quando foi apresentado aos alunos: os vídeos "A estória das coisas" e o "Rio+20: Desafios da sustentabilidade"; os pilares da sustentabilidade na palestra 1 e o vídeo "O homem capitalista" e a discussão sobre conflitos cognitivos decorrentes do uso desenfreado dos recursos naturais na palestra 2 .

Outro momento no qual identificamos o modelo de comunicação da experiência leiga foi na atividade da análise da imagem (Figura 2) pelos alunos proposta na palestra 1, visto que a discussão conduzida pela palestrante buscava que os alunos identificassem e discutissem implicações do desenvolvimento científico e tecnológico na sociedade e no ambiente a partir de seus conhecimentos adquiridos em suas experiências de vida.

Outro aspecto identificado foi uma maior aproximação da palestra 1 ao modelo de comunicação de participação pública, ou seja, aquele modelo que busca a participação dos cidadãos (FARES; NAVAS; MARANDINO, 2007). Isto não significa que na palestra 2 evidências deste modelo de comunicação não tenham sido identificadas. A busca pela participação dos alunos foi evidenciada quando a palestrante incentivou os mesmos a se posicionarem, no blog do projeto de extensão, sobre o que pode ser feito para que no futuro o fato de uma criança só ver uma árvore no museu não se torne realidade. Dessa forma, embora o blog do projeto de extensão não tenha sido construído em uma perspectiva mais política de participação em tomada de decisões sobre as aplicações da ciência e da tecnologia na sociedade, tornou-se um instrumento que possibilitou, com as postagens dos alunos, o exercício de participação pública. Além disso, este exercício de participação pode fazer emergir nos alunos um interesse e um engajamento em discussões que envolvam a tomada de decisão acerca da ciência e da tecnologia em suas relações com a sociedade.

Portanto, em síntese, as palestras 1 e 2, ao longo de seu desenvolvimento, não seguiram um único modelo de comunicação da ciência e da tecnologia, mas contemplaram aspectos característicos dos quatro modelos de comunicação discutidos, com maior predominância de aspectos relativos aos modelos de comunicação contextual, da experiência leiga e de participação pública. Nesse sentido, algumas implicações desses modelos de comunicação no desenvolvimento das palestras 1 e 2 para o letramento científico e tecnológico dos alunos puderam ser observadas. 
Divulgação científica: analisando modelos de comunicação da ciência e tecnologia e implicações para o letramento científico e tecnológico

O fato do modelo de comunicação déficit cognitivo não ter sido predominante nas duas palestras, pode ter contribuído para desmistificar um entendimento de ciência e de tecnologia como formas de conhecimentos elitizados e de cidadãos comuns que apenas necessitam das informações científicas e tecnológicas sem, necessariamente, agregar a estas um juízo de valor para uma avaliação crítica sobre as mesmas. A ideia de ciência e de tecnologia como construções humanas, sociais, coletivas e não dogmáticas, é condição necessária para o letramento científico e tecnológico dos alunos, visto que é esperado que uma pessoa letrada científica e tecnologicamente possa reconhecer, por exemplo, que o conhecimento científico é provisório, não elitista e sujeito a mudanças (FOUREZ, 1994).

Os momentos das palestras nos quais o modelo de comunicação contextual (FARES; NAVAS; MARANDINO, 2007) foi identificado podem ter contribuído para o letramento científico e tecnológico dos alunos na medida em que parecem: ter favorecido a compreensão de conhecimentos científicos e tecnológicos, visto que esta compreensão é facilitada quando tais conhecimentos são discutidos no âmbito de uma temática, o Complexo de Suape, que faz parte do contexto dos alunos; e ter subsidiado os alunos a lançarem mão dos conhecimentos científicos e tecnológicos compreendidos quando das tomadas de decisões ao longo de suas vidas, ou seja, em suas práticas sociais. Dessa forma, este modelo de comunicação parece ter contribuído para o letramento científico e tecnológico dos alunos à medida que buscou propiciar um entendimento de ciência e tecnologia como atividades sociais que influenciam a sociedade, como, por exemplo, nas discussões sobre o desenvolvimento do Complexo de Suape e os conflitos ambientais decorrentes desse desenvolvimento.

Quanto aos momentos das palestras nos quais o modelo da experiência leiga ficou evidente, na apresentação e na discussão do vídeo "A estória das coisas" e na atividade da análise da imagem (Figura 2) (palestra 1), e na apresentação e discussão do vídeo "O homem capitalista" (vídeo 2) e dos conflitos ambientais decorrentes da instalação e do funcionamento do Complexo de Suape (palestra 2), eles parecem ter contribuído para estimular uma reflexão pelos alunos sobre o consumo desenfreado em uma sociedade que segue o modelo de desenvolvimento capitalista e as consequências deste consumo, e sobre a relação custo/benefício do uso dos recursos naturais como matéria-prima dos diversos processos de produção industrial, destacando a necessidade de um modelo de desenvolvimento sustentável. Este exercício de reflexão dos alunos pode ter favorecido a emergência de uma visão crítica acerca do uso não sustentável de matérias-primas extraídas do meio ambiente para a produção científica e tecnológica. Além disso, estes momentos, caracterizados como modelo de comunicação da experiência leiga, podem ter 
Divulgação científica: analisando modelos de comunicação da ciência e tecnologia e implicações para o letramento científico e tecnológico

contribuído para o letramento científico e tecnológico dos alunos à medida que: propiciaram a compreensão das informações científicas e tecnológicas considerando os conhecimentos que os alunos têm em suas experiências cotidianas sobre os prejuízos que o desenvolvimento científico e tecnológico não planejado por causar para a sociedade e para o meio ambiente; e possibilitaram aos alunos a identificação de relações entre ciência, tecnologia e sociedade.

Contudo, é relevante mencionarmos que tanto a palestra 1 como a palestra 2 também buscaram promover aos alunos a compreensão das contribuições da ciência e da tecnologia na sociedade, embora este aspecto tenha ficado mais evidente na palestra 2. As discussões acerca do vídeo "Vídeo Institucional sobre o Porto de Suape" e dos programas desenvolvidos pela administração deste complexo portuário que buscam o desenvolvimento sustentável, mostraram evidências das contribuições da ciência e da tecnologia para o desenvolvimento do Estado de Pernambuco. Nesse sentido, não é objetivo das atividades de divulgação científica constitutivas do Projeto de Extensão "Divulgação científica em escolas públicas: uma ferramenta para o letramento científico e tecnológico" negar as contribuições da ciência e da tecnologia para a sociedade. Pelo contrário, é esperado o exercício de tomada de decisão pelos alunos de modo que eles considerem a relação custo/benefício acerca das aplicações e implicações do desenvolvimento científico e tecnológico na sociedade em que vivem.

Em relação aos momentos das palestras nos quais identificamos o modelo de comunicação de participação pública, quando a palestrante incentivou os alunos a se posicionarem no blog do projeto de extensão, parecem ter contribuído para o letramento científico e tecnológico dos alunos à medida que propiciou aos mesmos o entendimento de que a sociedade através de políticas públicas e pressões sociais pode exercer influência sobre, por exemplo, as aplicações da ciência e da tecnologia (FOUREZ, 1994).

Adicionalmente, a participação dos alunos no blog ao postarem suas opiniões acerca do fato de que no futuro uma criança veja uma árvore apenas no museu, pode ter contribuído para que eles desenvolvam o exercício de pensamento crítico e de tomada de decisão diante do modelo de desenvolvimento científico e tecnológico não sustentável.

Portanto, considerando as análises empreendidas neste estudo, entendemos que as atividades de divulgação científica por meio das palestras 1 e 2, seguindo os modelos de comunicação de ciência e tecnologia identificados, parecem se constituir como ferramenta para o letramento científico e tecnológico dos alunos envolvidos no projeto de extensão "Divulgação Científica em Escolas Públicas: uma ferramenta para o letramento científico e tecnológico", ao tempo em que contribuíram para: a compreensão de conhecimentos científicos e tecnológicos; os 
Divulgação científica: analisando modelos de comunicação da ciência e tecnologia e implicações para o letramento científico e tecnológico

alunos lançarem mão dos conhecimentos científicos e tecnológicos nas tomadas de decisões ao longo de suas vidas, ou seja, em suas práticas sociais; a identificação de relações entre ciência, tecnologia e sociedade; a compreensão de que as aplicações da ciência e da tecnologia também podem gerar problemas sociais e ambientais; o entendimento de que a sociedade através de políticas públicas e pressões sociais pode exercer influência sobre, por exemplo, aplicações da ciência e da tecnologia; o exercício de pensamento crítico e de tomada de decisão diante do modelo de desenvolvimento científico e tecnológico não sustentável

Ressaltamos que todos esses aspectos são característicos de um indivíduo letrado científica e tecnologicamente. É nesse sentido que vislumbramos o letramento científico e tecnológico de alunos das escolas públicas. E assumimos que este letramento está posto "como uma dimensão essencial da cultura de cidadania" (CACHAPUZ et al., 2005, p. 29). Portanto, entendemos que, em conjunto, os modelos de comunicação de ciência e tecnologia identificados nas atividades de divulgação científica, através das palestras 1 e 2 , instituíram tais atividades como uma ferramenta efetiva para o letramento científico e tecnológico dos alunos participantes do respectivo projeto de extensão.

\section{CONSIDERAÇÕES FINAIS}

Em uma sociedade culturalmente marcada pelos produtos da ciência e da tecnologia, ter seus cidadãos científica e tecnologicamente letrados é uma condição essencial para participação pública. Nessa direção, as análises empreendidas neste estudo apontaram que as atividades de divulgação científica desenvolvidas nas escolas, através de palestras, contribuíram para o letramento científico e tecnológico dos alunos assistidos pelo projeto de extensão "Divulgação científica em escolas públicas: uma ferramenta para o letramento científico e tecnológico". Portanto, pretendemos ampliar o alcance deste projeto de extensão para outras escolas públicas da região metropolitana do Recife-PE e planejarmos atividades de divulgação científica nas quais o modelo de comunicação de participação pública tenha um maior espaço em relação aos demais. 
Divulgação científica: analisando modelos de comunicação da ciência e tecnologia e implicações para o letramento científico e tecnológico

\section{AGRADECIMENTOS}

Agradecemos à PRAE (Pró-Reitoria de Atividades de Extensão da Universidade Federal de Pernambuco) e aos gestores, professores e alunos das escolas nas quais o projeto de extensão "Divulgação Científica em Escolas Públicas: uma ferramenta para o letramento científico e tecnológico" foi desenvolvido.

\section{REFERÊNCIAS}

BAUMGARTEN, M. O debate público de ciência e tecnologia: divulgação, difusão e popularização. Em: KERBAUY, M. T. M.; ANDRADE, T. H. N. de.; HAYASHI, C. R. M. (Org.). Ciência, Tecnologia e Sociedade no Brasil. Campinas, SP: Editora Alínea, 2012.

BAZZO, W. A. Ciência, Tecnologia e Sociedade: e o contexto da educação tecnológica. Florianópolis: Ed. da UFSC, 1998.

BECK, U. Sociedade de risco: rumo a uma outra modernidade. $2^{a}$ Ed. São Paulo: Editora 34, 2011.

BECK, U; GIDDENS, A.; LASH, S. Modernização reflexiva: política, tradição e estética na ordem social moderna. Portugal: Celta Editora, 2000.

BUSTAMANTE, J. A integração da ciência, tecnologia e sociedade: o grande desafio da educação no século XXI. Revista Educação Brasileira, v. 19, n. 39, p. 11-20, 1997.

CACHAPUZ, A.; GIL-PEREZ, D.; CARVALHO, A. M. P. de; PRAIA, J.; VILCHES, A. A necessária renovação do ensino das ciências. São Paulo: Cortez, 2005.

FARES, D. C.; NAVAS, A. M., MARANDINO, M. Qual a participação? Um enfoque CTS sobre os modelos de comunicação pública da ciência nos museus de ciência e tecnología. $\mathbf{X}$ Reunión de la Red de Popularización de la Ciencia y la Tecnología en América Latina y el Caribe (RED POP - UNESCO) y IV Taller "Ciencia, Comunicación y Sociedad". San José, Costa Rica, 2007. Disponível em: www.cientec.or.cr/pop/2007/BR-DjanaFares.pdf. Acesso em 30 de maio, 2016.

FERREIRA, L.N.A.; QUEIROZ, S. L. Textos de Divulgação Científica no Ensino de Ciências: uma revisão. Alexandria - Revista de Educação em Ciência e Tecnologia, v. 5, n. 1, p. 3-31, 2012.

FOUREZ, G. Alfabetización cientifica y tecnológica: acerca de las finalidades de la enseñanza de las ciências. $1^{\text {a }}$ ed. Ediciones Colihue, 1994. 
Divulgação científica: analisando modelos de comunicação da ciência e tecnologia e implicações para o letramento científico e tecnológico

GIL PÉREZ, D.; VILCHES, A. Contribuição da Educação secundária à formação de cidadãs e cidadãos para uma sociedade sustentável. In: MACEDO, B (org). Cultura científica: um direito de todos. Brasília: UNESCO Brasil, OREALC, MEC, MCT, 2003.

HERCULANO, S. Conflitos e natureza. Em: SILVA, T. A. A. da; GEHLEN, V. R. F. Conflitos socioambientais em Pernambuco. Recife: Fundação Joaquim Nabuco, Editora Massangana, 2013.

RESENDE, L. P.; ROTHBERG, D. Estudos CTS, comunicação e democracia digital. Em: HOFFMANN, W. A. M. (Org.). Ciência, tecnologia e sociedade: desafios para a construção do conhecimento. São Carlos: EDUFScar, 2011.

SANTOS, W. L. P. dos. Letramento em química, educação planetária e inclusão social. Revista Química Nova, v. 29, n. 3, p. 611-620, 2006.

SILVEIRA, K. A; BARROS, A. P. de. Conflitos socioambientais e participação social no complexo industrial de Suape. Em: SILVA, T. A. A. da; GEHLEN, V. R. F. Conflitos socioambientais em Pernambuco. Recife: Fundação Joaquim Nabuco, Editora Massangana, 2013. 